\title{
La analgesia peridural aumenta los requerimientos de ocitocina en el trabajo de parto, pero no aumenta las cesáreas
}

Epidural analgesia in association with duration of labor and mode of delivery: A quantitative review. Zhang J, Klebanoff MA, DerSimonian R. Am J Obstet Gynecol 1999; 970-7

\section{Objetivo}

Analizar cuantitativamente la literatura sobre los efectos de la analgesia peridural (AP) sobre la duración y la forma de terminación del parto.

\section{Fuentes y Selección de datos}

Se revisaron los estudios originales publicados en idioma inglés desde el año 1965 hasta diciembre de 1997 en Medline (palabras clave en inglés: epidural, labor, forceps, cesarean and delivery). También buscaron en las referencias de los mismos. Dos de los autores, de manera independiente, le asignaron un puntaje de calidad a cada artículo (de 1 a 5, siendo 5 los de máxima calidad). Se evaluaron los estudios que lograron los requerimientos mínimos. La síntesis de los datos se realizó de manera separada de acuerdo al diseño del estudio y la medición de los resultados; incluyendo la operación cesárea, el parto instrumental, la utilización de ocitocina y la duración del período de dilatación y del período expulsivo.

\section{Resultados}

Un total de siete ensayos clínicos aleatorizados y cinco estudios ob- servacionales cumplieron con los criterios mínimos. Para la síntesis de los datos se seleccionaron cuatro estudios de cada grupo. Ambos tipos de estudios mostraron que la AP aumenta al doble el riesgo de incrementar la necesidad de ocitocina. Los ensayos clínicos mostraron que la analgesia peridural no aumentó la incidencia de operación cesárea en todas sus indicaciones, incluyendo la distocia; ni aumentó la incidencia de parto instrumental. Los estudios observacionales, en cambio, mostraron que la incidencia de operación cesárea y de parto instrumental aumentó más de 4 veces. Si bien la mayoría de los trabajos mostraron un aumento en la duración del trabajo de parto en las mujeres con analgesia peridural, especialmente en el período expulsivo, los análisis estadísticos fueron inapropiados.

\section{Conclusiones}

La analgesia peridural, con bajas dosis de bupivacaina, podría aumentar las necesidades de ocitocina pero no la incidencia de operación cesárea.

\section{COMENTARIO}

Esta revisión intenta evaluar la evidencia publicada hasta el momento sobre la AP. Los estudios clínicos aleatorizados han sido difíciles de realizar dado que la AP se utiliza de manera corriente y ofrece una marcada disminución de dolor durante el trabajo de parto; asimismo no es posible realizar estudios doble ciego y las indicaciones de la operación cesárea o de los partos instrumentales son subjetivas y varían de institución a institución. Tampoco es sencillo objetivar si el aumento en la utilización de ocitocina en el grupo con AP se debe a una disminución de la contractilidad o a una habitual aplicación de la misma una vez iniciada esta anestesia. Algunas de las investigaciones que fueron seleccionadas en esta revisión constituyen estudios observacionales de partos espontáneos, diseñados específicamente para evaluar la relación existente entre la anestesia peridural y la operación cesárea. Estos mostraron que los índices de cesárea y de partos instrumentales no se modifican en las mujeres nulíparas con partos en presentación cefálica. Anteriormente se creía que el bloqueo motor provocaba una distocia, sin embargo recientes evidencias sugieren que este bloqueo no aumenta el riesgo del parto operatorio1. Con respecto a la duración del período expulsivo la mayoría de los estudios muestran una diferencia en las medias de duración del mismo. Dado que esta variable no se distribuye normalmente, la forma correcta de analizarla hubiera sido a traves de la medianas* y de test no paramétricos*.

Como en todo meta-análisis, uno de los sesgos principales es el sesgo de publicación, mediante el cual solo se analizan los estudios publicados, sin tomar en cuenta aquellos que no han llegado a la publicación. Asimismo la opinión utilizada de los dos autores en la clasificación del grado de calidad de los trabajos publicados también puede haber sido arbitraria. Con respecto a la homogeneidad de los distintos estudios incluídos en este metaanálisis, existen algunos datos importantes sobre los que existe considerable variabilidad, como por ejemplo las condiciones de ingreso de cada paciente en cada estudio, las indicaciones de ocitocina, las indicaciones de operación cesárea o el momento en que se ingresa en el período expulsivo. Por ello las conclusiones deben ser tomadas con cautela.

Como resumen esta revisión muestra que:

* La AP aumenta al doble la utilización de ocitocina (dato confiable)

* La AP no aumenta el riesgo de operación cesárea (probable)

* La AP podría aumentar levemente el riesgo de parto instrumental (no confiable)

* La AP prolonga el período expulsivo (no queda claro)

*Ver glosario

\section{Dr. Mario Sebastiani}

Servicio de Obstétricia . Hospital Italiano de Buenos Aires. 


\section{Control glucémico con dieta, sulfonilurea, metformina o insulina en pacientes con diabetes mellitus Tipo II}

Glycemic Control With Diet, Sulfonylurea, Metformin, or Insulin in Patients With Type 2 Diabetes Mellitus. Turner RC; Cull CA; Frighi V et al. ; for the UK Prospective Diabetes Study (UKPDS) Group. JAMA 1999;281:2005-12.

\section{Objetivo}

Evaluar la efectividad de distintas terapias para alcanzar los niveles ideales de control glucémico establecidos por la Asociación Americana de Diabetes (glucemia en ayunas menor de $140 \mathrm{mg} / \mathrm{dL}$ y hemog lobina glicosilada[HbA1c] menor de $7 \%$ ).

\section{Diseño}

Ensayo clínico aleatorizado y controlado realizado entre 1977 y 1997. Los pacientes fueron controlados cada tres meses hasta los nueve años luego del ingreso al estudio.

\section{Lugar}

Pacientes ambulatorios de 15 clínicas de diabetes del Reino Unido de Gran Bretaña.

\section{Pacientes}

Se incluyeron 4075 pacientes con diagnóstico reciente de diabetes tipo II reclutados en centros de atención primaria. Se consideró como criterio para el diagnóstico de diabetes la presencia de dos valores de glucemia en ayunas mayores de $108 \mathrm{mg} / \mathrm{dL}$. La edad de los pacientes osciló entre 25 y 65 años, y el $81 \%$ era de raza blanca. El nivel promedio de glucemia en ayunas al momento del diagnóstico era de $207 \mathrm{mg} / \mathrm{dL}$ y la concentración promedio de $\mathrm{HbA} 1 \mathrm{c}$ de $9.1 \%$.

\section{Intervención}

A todos los pacientes se les prescribió inicialmente una dieta hipograsa rica en fibra y carbohidratos. Después de tres meses de tratamiento se los dividió en tres categorías de acuerdo a la concentración media de glucemia evaluada en tres ocasiones separadas.

Los pacientes sintomáticos con concentraciones de glucemia en ayunas mayores a $270 \mathrm{mg} / \mathrm{dL}$ fueron aleatorizados a tratamiento intensivo con sulfonilurea o insulina; mientras que los obesos recibieron monoterapia con metformina.

Los pacientes sintomáticos con concentraciones de glucemia en ayunas entre 107 y $270 \mathrm{mg} / \mathrm{dL}$ fueron distribuidos de la misma manera que el grupo anterior, salvo un grupo que recibió solamente trata- miento no farmacologico. Los que presentaban glucemia en ayunas menores a $108 \mathrm{mg} / \mathrm{dL}$ fueron asignados exclusivamente a dieta. Si en controles posteriores su glucemia en ayunas era mayor o si aparecían síntomas de hiperglucemia, eran redistribuidos en los grupos mencionados previamente, a través de una aleatorización diferida.

El objetivo del tratamiento fue mantener niveles de glucemia en ayunas menores a $108 \mathrm{mg} / \mathrm{dL}$ con la terapia asignada. Existía la posibilidad de aumentar las dosis de sulfonilurea o metformina. El régimen inicial de insulina consistió en una dosis única de insulina de acción prolongada. Si la dosis diaria estaba por encima de 14 unidades se adicionaba insulina corriente al régimen usado. Aquellos pacientes que a pesar de la terapia combinada con sulfonilurea y metformina desarrollaron hiperglucemia fueron asignados a tratamiento con insulina.

\section{Medición de resultados}

Dosaje de glucemia en ayunas y HbA1c.

\section{Resultados}

Durante tres años de seguimiento, menos del $55 \%$ de los pacientes tratados con monoterapia pudieron alcanzar las metas terapéuticas. Sin embargo, comparando la terapia no farmacológica con la monoterapia, se vio que esta última duplica la proporción de pacientes que las logran alcanzar. La edad temprana al momento del diagnóstico de diabetes, la hipertrigliceridemia y la obesidad se relacionaron con mayor probabilidad de requerir terapia múltiple. Comparados con los que recibieron exclusivamente dieta, los pacientes que recibieron tratamiento intensivo tuvieron menor probabilidad de requerir terapia adicional para alcanzar niveles de HbA1c menores de 7\% (OR 0,55; IC95\%, 0,43 a 0,69; p $<0.001$ ) y para lograr una concentración de glucemia en ayunas menor a $140 \mathrm{mg} / \mathrm{dL}($ OR 0,$34 ;$ IC $95 \% 0,27$ a $0.44 ; \mathrm{p}<0.001)$.

\section{Conclusiones}

Las metas glucémicas sugeridas por la AAD sólo pueden ser alcanzadas por una minoría de los pacientes con monoterapia.

Fuente de financiamiento: Novo Nordisk; Bayer; Bristol Myers; Hoechst Marion Roussel; Eli Lilly \& Co; Lipha; Farmitalia Carlo Erba.

\section{COMENTARIO}

Diversas publicaciones han demostrado las ventajas del tratamiento intensivo de la diabetes. Esta evidencia sustentó inicialmente el tratamiento intensivo de la diabetes tipo I 1 , existiendo hoy en día algunos trabajos que tambien avala este tipo de estrategia para los pacientes con diabetes tipo II ${ }^{2-3}$.

Respecto de este trabajo, todas las estrategias de monoterapia incrementaron dos o tres veces la proporción de pacientes que Lograron las metas terapéuticas, en comparación con los tratados no farmacologicamente. Sin embargo, luego de tres años del diagnóstico, aproximadamente el $50 \%$ de los pacientes necesitaron más de un agente farmacológico (a los nueve años, el 75\% requirió terapia múltiple).

La eficiencia de la adición temprana de metformina al tratamiento máximo con sulfonilureas fue demostrada después de tres años y aumentó el porcentaje de individuos que lograron el control glucémico. No obstante, la mayoría requirió a largo plazo la adición de insulina. Se vio que existían ciertas variables que se relacionaban a la necesidad de requerir terapia múltiple; concluyéndose que la edad temprana al momento del diagnóstico, la obesidad, la hipertrigliceridemia y la hiperglu- cemia basal fueron factores predictivos del fracaso de la monoterapia. Si bien este trabajo incluyó una muestra numerosa de pacientes y se los siguió durante un período adecuado, es de destacar que la glucemia promedio en estos pacientes y los criterios usados para iniciar tratamiento son muy diferentes a los actualmente manejados con una población similar. Además, los criterios utilizados para el diagnóstico de diabetes (glucemia en ayunas mayor a $108 \mathrm{mg} / \mathrm{dL}$ en dos o más oportunidades) son diferentes a los actuales para el diagnóstico, a saber: 1) síntomas clínicos como poliuria, polidipsia, polifagia o pérdida de peso acompañados de glucemias $\geq 200 \mathrm{mg} / \mathrm{dL}$ en cualquier momento; 2) glucemia en ayunas $\geq$ a $126 \mathrm{mg} / \mathrm{dL} ; 3$ ) glucemia $\geq 200 \mathrm{mg} / \mathrm{dL}$ luego de dos horas de una carga oral con $75 \mathrm{~g}$ de glucosa (cualquiera de estos criterios debe ser documentado en dos ocasiones en días diferente).

De todas maneras, si bien este estudio nos demuestra que la mayoría de los diabéticos adultos requerirán terapias múltiples a largo plazo, no queda claro aún cuál o cuales son los intervalos a tomar en cuenta para incrementar la intensidad del tratamiento en pos de lograr los objetivos planteados.

Dra. Roxana Pilotti Hospital Ramos Mejía de Buenos Aires.

\section{Referencias}

1. Effect of intensive blood-glucose control with metformin on complications in overwright patients wth type 2 diabetes. The Lancet 1998; $352: 854-865$.

2. The DCCT Research Group. The effect of intensive tratment of Diabetes on the development and porgresion of long term complications in insulin dependent diabetes mellitus. N Eng J Med.1993; 329:997-86.

3. Vijan S, Stevens DL, Herman WH, Funnell MM, Standiford CJ. Screeninig, prevention, counseling, and treatment for the complications of type 2 diabetes mellitus. Putting evidence into practice. J Gen Intern Med 1997;12:567-80 